\title{
Superliga feminina de voleibol: análise das ações para obtenção de pontos na temporada de 2015-2016
}

Women's volleyball super league: analysis of actions to obtain points in the 2015-2016 season

\author{
Ana Flávia Inacio Sabino ${ }^{1}$ \\ Dayana Chaves Franco ${ }^{1}$ \\ Thiago Ferreira de Sousa ${ }^{1,2, *}$
}

\section{Resumo:}

Objetivo: Comparar o desempenho entre as equipes que competiram na Superliga Feminina de Voleibol, na temporada 2015-2016, nas ações relacionadas à obtenção da pontuação (ataque e saque). Métodos: Foi realizado um estudo documental, por meio do levantamento das informações nas bases de dados da competição. Foram extraídas as informações da etapa classificatória da competição das 12 equipes participantes, perfazendo um total de 22 rodadas, em relação a quantidade de ataques e saques, os números de erros de ataque e saque e números de pontos de ataque e saque. Realizou-se o cálculo da eficácia do ataque e saque. As comparações entre as ações de ataque e defesa, entre as equipes, foram realizadas pelo teste Anova, oneway, complementadas pelo teste post hoc de Tukey. O nível de significância foi de 5\%. Resultados: Foram analisados 132 jogos, sendo jogos de ida e volta. De modo geral, a eficácia no ataque foi de $28,6 \%$ e a eficácia no saque foi de $-4,1 \%$. Observou-se que a média de eficácia no ataque foi maior para a equipe do Rexona Ades (35,6\%). As equipes de Valinhos Country e São Bernardo Vôlei apresentaram as menores médias de pontos de ataque. Conclusão: Notou-se discrepância nas médias de pontos e erros de ataque e saque para obtenção de pontos entre as equipes, sendo que a equipe que foi campeã da competição (Rexona Ades) mostrou melhor eficácia nas ações de ataque na etapa de classificação.

Palavras-chave: desempenho psicomotor, desempenho atlético, voleibol.

\begin{abstract}
Objective: To compare performance among teams that competed in the Feminine Volleyball Superliga in the 2015-2016 season in actions related obtaining the score (attack and serve). Methods: A documentary study was carried out, by means of the survey of the information in the databases of the competition. The information about the competition's qualifying stage was extracted from the 12 participating teams, from 22 rounds, in relation to the number of attacks and volleyball kicks, the numbers of attack and serve errors, and numbers of attack and serve points. The effectiveness of the attack and volleyball kicks was calculated. The comparisons between the attack and volleyball kicks actions, among the teams, were performed by the Anova test, one way, complemented by the post hoc test of Tukey. The level of significance was 5\%. Results: Were analyzed 132 games, being round-trip games. In general, the attack effectiveness was $28.6 \%$ and the effectiveness of volleyball kicks was $4.1 \%$. Was observed that the mean effectiveness in the attack was higher for the Rexona Ades team (35.6\%). The Valinhos Country and São Bernardo Volleyball teams had the lowest average points of attack. Conclusion: Was noticed a discrepancy in the average of points and errors of attack and volleyball kicks to obtain points between the teams, being that the team that was champion of the competition (Rexona/Ades) showed better effectiveness in the actions of attack in the classification stage.
\end{abstract}

Keywords: athletic performance, psychomotor performance, volleyball.
Afiliação dos autores

${ }^{1}$ Universidade Federal do Triângulo Mineiro, Uberaba, Minas Gerais, Brasil.

${ }^{2}$ Universidade Federal do Recôncavo da Bahia, Amargosa, Bahia, Brasil

*Autor correspondente

Universidade Federal do Recôncavo da Bahia. Centro de Formação de Professores. Avenida Nestor de Mello Pita, o 535 - Número da caixa postal: 64 , Centro, CEP: $45.300-000$, Amargosa, BA, Brasil,

e-mail:

tfsousa_thiago@yahoo.com.br

Conflito de interesses

Os autores declararam não haver conflito de interesses.

Processo de arbitragem

Recebido: 29/11/2018 Aprovado: 08/01/2019 


\section{Introdução}

O esporte compõe um importante elemento cultural $^{1}$, seja como pratica no lazer ou voltada para o desempenho. O esporte de rendimento, também referenciado como esporte de competição é praticado obedecendo os códigos e regras estabelecidos por entidades internacionais, do qual objetiva resultados positivos, vitórias, alcance de recordes, títulos esportivos e prêmios financeiros ${ }^{2}$.

Dentre os esportes de desempenho, o voleibol, caracterizado como um jogo dinâmico apresenta a característica de confronto entre duas equipes sem contato físico, com o objetivo de colocar a bola no chão da quadra adversária ${ }^{3}$, não sendo permitido segurar a bola durante a partida, assim como tocá-la duas vezes consecutivamente ${ }^{4}$. Essa modalidade tem sido também praticada no momento do lazer por adolescentes ${ }^{5}$, universitários ${ }^{6}$ e adultos ${ }^{7}$ brasileiros.

As ações voltadas à obtenção de pontos nessa modalidade são o ataque e o saque (reposição da bola em quadra) ${ }^{8}$. Tais movimentos são empregados durante o jogo de forma técnica e tática, buscando o alcance dos pontos para as vitórias. $\mathrm{O}$ ataque é um dos fundamentos mais precisos para finalização de um rally, e com a obtenção de ponto para a equipe que atacou configura-se como eficácia dessa ação. O saque no voleibol é um fundamento que inicia a jogada, podendo ser empregado estrategicamente como forma de ataque, visando a obtenção de ponto direto ${ }^{9}$.

Diante disso, a caracterização dessas ações nos jogos oficiais, em equipes de alto nível, pode contribuir para o entendimento do papel do ataque e saque na competição, bem como orientar as futuras equipes sobre essas ações para a obtenção de pontos. $\mathrm{Na}$ literatura nota-se a necessidade de estudos voltados a apresentação da eficácia das ações ofensivas do voleibol feminino brasileiro de alto nível, bem como apresentar o desempenho do ataque e saque especificamente em função das equipes ${ }^{10}$. Encontram-se alguns trabalhos que investigaram as categorias de base e sobre Superliga Masculina de voleibol $\left.\right|^{9,11-13}$

Sendo assim, tendo em vista a relevância da competição da Superliga Feminina de Voleibol no Brasil, que apresenta elevado status no cenário competitivo brasileiro, o objetivo deste estudo foi comparar o desempenho entre as equipes que competiram nessa competição, na temporada 2015-2016, nas ações relacionadas à obtenção da pontuação (ataque e saque).

\section{Métodos}

Esta pesquisa de caráter quantitativo, descritiva e documental ${ }^{14}$, teve como base as informações provenientes do scout das 12 equipes da Superliga Brasileira Feminina de Vôlei, divisão Série $A$, que disputaram a competição durante a temporada de 2015/2016, disponibilizado no site da Superliga Brasileira Feminina de Vôlei (http://superliga.cbv.com.br/1516/index.php/2014-10-24-18-24-03/tabela-de-jogos/tabela-dejogos-2016). As informações, que são de acesso público, foram extraídas nesse endereço nos meses de agosto e setembro de 2017.

As equipes que participaram dessa temporada foram: Dentil Praia Clube, São Cristóvão, Vôlei Bauru, Osasco Vôlei Nestlé, Rio do Sul Equibrasil, Camponesa Minas, Rexona/Ades, Valinhos Country, SESI- SP, São Bernardo, Brasília Vôlei e Pinheiros/Klar. As informações que foram extraídas desses relatórios e representam as variáveis dependentes deste estudo, considerando apenas as 22 rodadas da fase classificatória foram: quantidade de ataques realizados, erros de ataque, pontos de ataque, quantidade de saque, erros de saque e pontos de saque. Com base essas informações foram elaboradas outras duas variáveis dependentes sendo: a eficácia do ataque (êxito com o ponto obtido) e eficácia do saque (êxito com o ponto obtido). A eficácia foi calculada pela seguinte equação: diferença entre os pontos obtidos e os erros obtidos nas ações dividido pela quantidade total da ação, multiplicado por 100 .

Os dados dos relatórios foram tabulados em planilha do Microsoft Office Excel, versão 2017, considerando a rodada da competição e a equipe. As análises foram realizadas no programa estatístico SPSS, versão 24.0. Foram realizadas análises univariadas para a verificação da normalidade dos dados, via teste de KolmogorovSmirnov. Observou-se 0 atendimento de normalidade, sendo assim foram realizadas análises descritivas de média, desvio padrão, mínimo e máximo valores. Para a comparação entre as 12 equipes foi realizado o teste ANOVA oneway e para a verificação dos grupos diferentes foi utilizado o teste post hoc Tukey. O nível de significância adotado foi de $5 \%$.

\section{Resultados}

Das informações disponibilizadas no site da competição foi observada a ausência dos resultados das equipes do Praia Clube e SESI-SP na rodada 17. As características gerais das ações referentes a obtenção de pontos são apresentadas na Tabela 1. Notou-se que a média de eficácia do ataque foi de $28,6 \%$ e a eficácia do saque foi de $-4,1 \%$. As médias dos erros de ataque e saque foram, aproximadamente, 10 e 7 , respectivamente.

Tabela 1

Análise descritiva das ações de ataque e saque das equipes da Superliga Feminina de Voleibol na temporada de 2015-2016.

\begin{tabular}{lcccccc}
\hline \multicolumn{1}{c}{ Variáveis } & $\mathrm{n}^{*}$ & Média & $\begin{array}{c}\text { Desvio } \\
\text { Padrão }\end{array}$ & Mínimo & Máximo \\
\hline № de ataques & 262 & 124,6 & 29,4 & 76 & 209 \\
№ de erros de ataque & 262 & 10,5 & 4,8 & 2 & 24 \\
№ de pontos de ataque & 262 & 45,8 & 12,1 & 13 & 77 \\
\% Eficácia pontos de ataque & 262 & 28,6 & 8,1 & 5,3 & 50,5 \\
№ de saques & 262 & 79,6 & 18,5 & 36 & 124 \\
№ de erro de saques & 262 & 7,3 & 7,2 & 1 & 112 \\
№ de pontos de saque & 262 & 4,1 & 2,4 & 0 & 10 \\
\% Eficácia de pontos de saque & 262 & $-4,1$ & 7,7 & $-100,0$ & 9,3 \\
\hline №: Número; *Q Quantidade de observações considerando & 12 equipes em 22
\end{tabular}

rodadas, com o missingde 2 equipes na rodada 17.

$\mathrm{Na}$ Tabela 2 são apresentadas as comparações entre as equipes quanto as ações relacionadas ao ataque e saque.A equipe Rio do Sul Equibrasil apresentou maior média de erros de ataque quando comparado as equipes Dentil Praia Clube, Vôlei Bauru, Osasco Vôlei Nestlé, Rexona/Ades e Valinhos Country. As equipes de Valinhos Country e São Bernardo Vôlei apresentaram as menores médias de pontos de ataque. A equipe do Rexona Ades apresentou maior média de pontos de saque, quando comparado as equipes Valinhos Country, São Bernardo Vôlei e Pinheiros/Klar. A equipe que apresentou maior média de eficácia de ataque foi a Rexona Ades, com média de $35,6 \%$.

\section{Discussão}

Neste estudo observou-se que a equipe RexonaAdes apresentou maior média de eficácia no ataque e não houve diferença na eficácia no saque. Além disso, a equipe Rio do Sul Equibrasil foi a que apresentou maior média de erros de ataque eas equipes de Valinhos Country e São Bernardo Vôlei apresentaram as menores médias de pontos de ataque. A equipe do RexonaAdes apresentou maior média de pontos de saque, quando comparado às equipes Valinhos Country, São Bernardo Vôlei e Pinheiros/Klar.

Notou-se que todas as equipes apresentaram média de pontos de ataque, com valores superiores a 40 , exceto as equipes do Vôlei Bauru, Valinhos Country e São Bernardo Vôlei. $\mathrm{O}$ ataque emerge como a ação de jogo com maior poder explicativo sobre o resultado do jogo ${ }^{11}$, especialmente por atletas de posições da zona de ataque ${ }^{15}$. Observou-se que a equipe que apresentou maior média de eficácia no ataque, durante a etapa classificatória, foi campeã da temporada, a Rexona Antes, com média de 35,6\%. Pode-se inferir que o bom desempenho nas ações de obtenção de pontos durante a etapa classificatória favoreceu a equipe na etapa de eliminatória. Além disso, no voleibol as ações de ataque têm a maior possibilidade de gerar um ponto em relação às outras ações de defesa, tais como a recepção e o levantamento, no entanto, um ataque terá uma maior probabilidade de sucesso se for precedido por um bom levantamento, bem como uma recepcão de sucesso, $0^{9,10}$

A eficácia do ataque consiste em ação com troca de passes entre as jogadoras para que a última atleta que estiver com a posse da bola realize a ação final, com a ocorrência de ponto, sendo a ação de efetivar o ponto superior a quantidade de erros dessas tentativas. A não observância das informações referentes a eficácia no passe representa um potencial limitador das características referentes ao ataque, ao caracterizar apenas 
os pontos e erros do ataque. Por outro lado, é possível inferir em atletas de alto nível que a eficácia desse fundamento pode estar relacionada a realização de ações de recepção e levantamento com eficiência, mediante condutas de ajustamento corporal e tomadas de decisões ${ }^{16}$. Porém, esses detalhamentos de informações nos scouts dessa competição não permitem melhor analisar a relevância da eficiência sobre o desempenho técnico e tático das equipes.

Tabela 2

Comparação das médias (desvio padrão) das características referentes as ações de ataque e saque entre as equipes da Superliga Feminina de Voleibol na temporada 2015-2016.

\begin{tabular}{|c|c|c|c|c|c|c|c|c|c|c|c|c|c|c|}
\hline \multirow[b]{2}{*}{ Variáveis } & \multicolumn{14}{|c|}{ Equipes } \\
\hline & $\begin{array}{l}\text { № de } \\
\text { rodadas }\end{array}$ & DPC & SC & VB & OVN & RSE & $\mathrm{CM}$ & RA & VC & SSP & SBV & BV & PK & $\mathbf{p}^{*}$ \\
\hline $\begin{array}{c}\text { № de } \\
\text { ataques }\end{array}$ & 22 & $\begin{array}{l}123,5 \\
(30,9)\end{array}$ & $\begin{array}{l}133,4 \\
(28,3)\end{array}$ & $\begin{array}{l}128,8 \\
(34,3)\end{array}$ & $\begin{array}{l}114,9 \\
(25,4)\end{array}$ & $\begin{array}{l}134,8 \\
(30,2)\end{array}$ & $\begin{array}{l}129,1 \\
(28,1)\end{array}$ & $\begin{array}{l}114,7 \\
(29,4)\end{array}$ & $\begin{array}{l}114,3 \\
(28,5)\end{array}$ & $\begin{array}{l}137,9 \\
(36,3)\end{array}$ & $\begin{array}{l}109,6 \\
(19,5)\end{array}$ & $\begin{array}{l}130,2 \\
(28,6)\end{array}$ & $\begin{array}{l}124,7 \\
(21,3)\end{array}$ & 0,01 \\
\hline $\begin{array}{l}\text { № de } \\
\text { erros de } \\
\text { ataque }\end{array}$ & 22 & $\begin{array}{c}8,6 \\
(3,9)^{\mathrm{a}}\end{array}$ & $\begin{array}{c}10,2 \\
(3,5)^{a, b}\end{array}$ & $9,4(4,0)^{a}$ & $\begin{array}{c}9,0 \\
(3,3)^{\mathrm{a}}\end{array}$ & $\begin{array}{c}13,8 \\
(5,1)^{b}\end{array}$ & $\begin{array}{c}11,1 \\
(4,6)^{a, b}\end{array}$ & $\begin{array}{c}8,3 \\
(3,7)^{\mathrm{a}}\end{array}$ & $\begin{array}{c}9,6 \\
(4,2)^{\mathrm{a}}\end{array}$ & $\begin{array}{c}10,4 \\
(4,5)^{\mathrm{a}, \mathrm{b}}\end{array}$ & $\begin{array}{c}11,4 \\
(3,7)^{a, b}\end{array}$ & $\begin{array}{c}12,4 \\
(4,3)^{a, b}\end{array}$ & $\begin{array}{c}12,0 \\
(4,6)^{a, b}\end{array}$ & $<0,01$ \\
\hline $\begin{array}{c}\text { № de } \\
\text { pontos de } \\
\text { ataque } \\
\%\end{array}$ & 22 & $\begin{array}{c}48,8 \\
(8,9)^{c}\end{array}$ & $\begin{array}{c}47,5 \\
(11,0)^{\mathrm{c}}\end{array}$ & $\begin{array}{c}43,3 \\
(12,8)^{b, c}\end{array}$ & $\begin{array}{l}47,8 \\
(9,5)^{\mathrm{c}}\end{array}$ & $\begin{array}{c}53,5 \\
(11,7)^{\mathrm{c}}\end{array}$ & $\begin{array}{l}51,9 \\
(9,6)^{\mathrm{c}}\end{array}$ & $\begin{array}{l}48,0 \\
(8,6)^{c}\end{array}$ & $\begin{array}{c}32,4 \\
(10,7)^{\mathrm{a}}\end{array}$ & $\begin{array}{c}46,6 \\
(15,9)^{\mathrm{c}}\end{array}$ & $\begin{array}{c}34,0 \\
(9,5)^{a, b}\end{array}$ & $\begin{array}{l}48,5 \\
(8,5)^{\mathrm{c}}\end{array}$ & $\begin{array}{l}47,8 \\
(9,2)^{\mathrm{c}}\end{array}$ & $<0,01$ \\
\hline $\begin{array}{l}\text { Eficácia } \\
\text { pontos de } \\
\text { ataque }\end{array}$ & 22 & $\begin{array}{c}33,7 \\
(7,8)^{\mathrm{d}, \mathrm{e}}\end{array}$ & $\begin{array}{c}27,9 \\
(5,4)^{\mathrm{c,d}}\end{array}$ & $\begin{array}{c}26,6 \\
(7,0)^{\mathrm{b}, \mathrm{c}}\end{array}$ & $\begin{array}{l}34,4 \\
(6,2)^{\mathrm{d}, \mathrm{e}}\end{array}$ & $\begin{array}{c}29,8 \\
(6,2)^{\mathrm{c}, \mathrm{d}, \mathrm{e}}\end{array}$ & $\begin{array}{c}32,1 \\
(6,1)^{\mathrm{c}, \mathrm{d}, \mathrm{e}}\end{array}$ & $\begin{array}{c}35,6 \\
(5,1)^{\mathrm{e}}\end{array}$ & $\begin{array}{c}19,6 \\
(6,7)^{a}\end{array}$ & $\begin{array}{c}26,0 \\
(6,8)^{\mathrm{a}, \mathrm{b}, \mathrm{c}}\end{array}$ & $\begin{array}{c}20,4 \\
(7,8)^{a, b}\end{array}$ & $\begin{array}{c}28,4 \\
(6,3)^{c, d}\end{array}$ & $\begin{array}{c}29,0 \\
(7,2)^{\mathrm{c,d}}\end{array}$ & $<0,01$ \\
\hline $\begin{array}{c}\text { № de } \\
\text { saques }\end{array}$ & 22 & $\begin{array}{c}84,8 \\
(15,5)^{\mathrm{b}}\end{array}$ & $\begin{array}{c}82,2 \\
(19,2)^{b}\end{array}$ & $\begin{array}{c}75,6 \\
(19,2)^{a, b}\end{array}$ & $\begin{array}{c}82,3 \\
(15,1)^{b}\end{array}$ & $\begin{array}{c}86,6 \\
(18,1)^{b}\end{array}$ & $\begin{array}{c}86,6 \\
(15,5)^{\mathrm{b}}\end{array}$ & $\begin{array}{c}83,9 \\
(13,6)^{b}\end{array}$ & $\begin{array}{c}62,1 \\
(17,9)^{\mathrm{a}}\end{array}$ & $\begin{array}{c}86,3 \\
(21,4)^{b}\end{array}$ & $\begin{array}{c}62,3 \\
(14,7)^{\mathrm{a}}\end{array}$ & $\begin{array}{c}83,9 \\
(15,8)^{b}\end{array}$ & $\begin{array}{c}79,3 \\
(13,6)^{b}\end{array}$ & $<0,01$ \\
\hline $\begin{array}{l}\text { № de } \\
\text { erro de } \\
\text { saques }\end{array}$ & 22 & $\begin{array}{c}6,2 \\
(2,1)\end{array}$ & $\begin{array}{c}6,4 \\
(1,9)\end{array}$ & $8,1(3,3)$ & $\begin{array}{c}8,9 \\
(2,8)\end{array}$ & $\begin{array}{c}8,0 \\
(3,3)\end{array}$ & $\begin{array}{c}8,4 \\
(3,8)\end{array}$ & $\begin{array}{c}5,7 \\
(2,6)\end{array}$ & $\begin{array}{c}5,0 \\
(2,3)\end{array}$ & $\begin{array}{c}10,2 \\
(23,4)\end{array}$ & $\begin{array}{c}7,6 \\
(3,2)\end{array}$ & $\begin{array}{c}6,1 \\
(3,3)\end{array}$ & $\begin{array}{c}7,0 \\
(2,9)\end{array}$ & 0,49 \\
\hline $\begin{array}{c}\text { № de } \\
\text { pontos de } \\
\text { saque } \\
\%\end{array}$ & 22 & $\begin{array}{c}4,6 \\
(1,9)^{a, b}\end{array}$ & $\begin{array}{c}3,8 \\
(2,4)^{a, b}\end{array}$ & $\begin{array}{c}3,7 \\
(2,4)^{a, b}\end{array}$ & $\begin{array}{c}4,4 \\
(2,4)^{\mathrm{a}, \mathrm{b}}\end{array}$ & $\begin{array}{c}4,7 \\
(2,4)^{a, b}\end{array}$ & $\begin{array}{c}4,4 \\
(2,0)^{\mathrm{a}, \mathrm{b}}\end{array}$ & $\begin{array}{c}5,8 \\
(2,3)^{b}\end{array}$ & $\begin{array}{c}2,9 \\
(2,3)^{\mathrm{a}}\end{array}$ & $\begin{array}{c}4,4 \\
(2,7)^{\mathrm{a}, \mathrm{b}}\end{array}$ & $\begin{array}{c}3,2 \\
(2,1)^{\mathrm{a}}\end{array}$ & $\begin{array}{c}4,2 \\
(1,9)^{a, b}\end{array}$ & $\begin{array}{c}3,4 \\
(2,5)^{a}\end{array}$ & $<0,01$ \\
\hline $\begin{array}{l}\text { Eficácia } \\
\text { de pontos } \\
\text { de saque }\end{array}$ & 22 & $\begin{array}{l}-1,7 \\
(2,6)\end{array}$ & $\begin{array}{l}-3,0 \\
(3,8)\end{array}$ & $-6,1(5,4)$ & $\begin{array}{l}-5,4 \\
(5,5)\end{array}$ & $\begin{array}{l}-3,7 \\
(4,3)\end{array}$ & $\begin{array}{c}-4,5 \\
(5,1)\end{array}$ & $\begin{array}{c}0,1 \\
(4,0)\end{array}$ & $\begin{array}{l}-4,0 \\
(5,7)\end{array}$ & $\begin{array}{c}-5,7 \\
(21,9)\end{array}$ & $\begin{array}{l}-7,5 \\
(4,8)\end{array}$ & $\begin{array}{l}-2,2 \\
(5,1)\end{array}$ & $\begin{array}{l}-4,7 \\
(3,4)\end{array}$ & 0,08 \\
\hline
\end{tabular}

DPC: Dentil Praia Clube; SC: São Cristóvão; VB: Vôlei Bauru; OVN: Osasco Vôlei Nestlé; RSE: Rio do Sul Equibrasil; CM: Camponesa Minas; RA: Rexona/Ades; VC:

Valinhos Country; SSP: SESI- SP; SB: São Bernardo Vôlei; BV: Brasília Vôlei; PK: Pinheiros/Klar.

${ }^{*} \mathrm{p}$ valor do teste ANOVA oneway. Letras diferentes indicaram diferenças entre as médias, de acordo com o teste post hoc de Tukey.

A eficácia no saque está ligada a ação de obter pontos com a ação de iniciar uma jogada. Dessa forma, o saque é um importante instrumento para obtenção de ponto direto ${ }^{13}$. Neste estudo não foram observadas diferenças na eficácia no saque entre as equipes. Porém, foi possível observar que de modo geral e também em função das equipes, exceto para a equipe que foi campeã, a Rexona Ades, a eficácia foi negativa. Esses resultados indicam uma maior quantidade de erros de saque, provavelmente pela tentativa de obtenção de pontos com essa ação, mediante o uso de formas de saque com viés de ataque, como o saque em suspensão potente ${ }^{12}$

Acredita-se que a ação do saque está condicionada ao momentodo jogo. Tendo em vista que o saque consiste no lançamento, ou batida na bola de início do rally, o momentodapartida poderá determinar o melhor momento de ocorrência de um saque mais ofensivo, visando dificultar o sistema de recepção da equipe adversária ${ }^{12}$. Além disso, pode ser levado em consideração, como por exemplo, a condição emocional da equipe adversária em dado momento do jogo, sendo em certas ocasiões mais relevante que a capacidade motora ou técnica do jogador que realiza a técnica de saque ${ }^{17}$

A média de erro de ataque na etapa classificatória foi de 10,5 e a média de erro de saque foi 7,3 . Observou-se que a equipe Rio do Sul Equibrasil foi a que apresentou maior média de erros de ataque eas equipes de Valinhos Country e São Bernardo Vôlei apresentaram as menores médias de pontos de ataque, e essas duas últimas equipes foram rebaixadas nessa temporada para a Superliga Brasileira Feminina de Vôlei Série B. As equipes que normalmente perdem os sets são aquelas que apresentam maiores quantidades de erros de ataque, bem como apresentam menor performance no ataque e saque ${ }^{11}$.

Neste estudo, as limitações estão relacionadas com a possibilidade de viés decorrente da base de dados, em virtude de possíveis erros de digitação, bem como a ausência de resultados, conforme observado pela falta de informações de duas equipes na $17^{\mathrm{a}}$ rodada. No entanto, 0 uso de análise de dados secundários permite o uso de informações com maior agilidade, como empregado em análises referentes ao orçamento ${ }^{18} \mathrm{e}$ informações sobre a saúde dos brasileiros ${ }^{19}$, e favorecem a diminuição de gastos com a realização de observações das partidas. Por outro lado, este estudo destaca as características comparativas entre as equipes participantes na temporada 2015-2016, da liga de voleibol, de alto nível feminina brasileira, e assim permite analisar o desempenho na etapa de classificação, que representa momento de maior estabilidade das equipes e de menor pressão inicial da eliminação.

\section{Conclusão}

Conclui-se que houve divergências entre a eficácia no ataque, com maior média para a equipe que foi campeã da competição (Rexona Ades), além disso, essa mesma equipe foi a que apresentou durante a etapa classificatória, menor média de erros de ataque e saque, e maior média de pontos de saque. Não houve diferenças nas médias de eficácia no saque, sendo esses valores negativos para a maioria das equipes. Sugere-se a realização de outros estudos que possam complementar as informações acerca das ações de defesa, bem como a análise desagregada para cada atleta durante a competição.

\section{Referências}

1. Betti M. A janela de Vidro: Esporte, Televisão e Educação Física. $3^{\underline{a}}$ ed. Campinas. Editora Papirus, 1997.

2. Tubino MJG. Estudos brasileiros sobre o esporte:ênfase no esporteeducação. Maringá. EditoraEduem, 2010, p.43.

3. Haiachi MC, Fernandes Filho J. Análise de saltos e rally no confronto entre Brasil e Itália nos Jogos Olímpicos de Atenas 2004. Ação \&Movimento:educação física e desportos2011;3(1):16-20.

4. Ugrinowitsch $\mathrm{H}$, et al. Transition I efficiency and victory in volleyball matches. Motriz: Revista de Educação Física2014;20(1):42-6.

5. Mendonça G, Cheng LA, Farias Junior JC. Padrões de prática de atividade física em adolescentes de um município da região Nordeste do Brasil. Ciência \& Saúde Coletiva2018;23(7):2443-51.

6. Sousa TF. Atividades físicas praticadas no lazer por universitários de uma instituição pública do nordeste do Brasil. RevistaBrasileira de Atividade Física e Saúde2012;17(2):125-31.

7. Lima DF, Lima LA, Silva MP. Tendências temporais dos tipos principais de exercício físico e esporte praticados no lazer na cidade de Curitiba, Brasil: 2006-2014. Revista Brasileirade Ciência e Movimento2017-25(3):98-105.

8. Weishoff, P. Ataque. In: Shondell DS; Reynaud C. A bíblia do treinador de voleibol. Porto Alegre: Artmed, 2005, p. 203-28.

9. Gil Arias A,et al. Análisis de la eficacia del saque de voleibol en categoría de formación. Revista Internacional de Medicina y Ciencias de la Actividad Física y el Deporte 2011;11(44):721-37.

10. Costa GCT, Barbosa RV, Gama Filho JG. A modulação do ataque no voleibol de alto nível: o caso da Superliga feminina 2011-2012. Revista da Educação Física/UEM2013;24(4):545-57.

11. MarcelinoR, et al. Estudo dos indicadores de rendimento em voleibol em função do resultado do set. Revista Brasileira de Educação Física e Esporte 2010;24(1):69-78.

12. Costa GCT, Mesquita I, Greco PJ, Ferreira NN, Moraes JC. Relação saque, recepção e ataque no voleibol juvenil masculino. Motriz: Revista de EducaçãoFísica2011;17(1):11-8. 
13. Castro HO, Cavalli I, Silva CJA, Greco PJ. Interação no Curso das Ações de Saque e Bloqueio no Voleibol Juvenil. Revista da Faculdade de Educação Física da UNICAMP2014;12(3):34-54

14. Gil AC. Como elaborar projetos de pesquisa. $4^{\text {a }}$ ed. São Paulo: Editora Atlas S.A., 2002

15. Marques Junior NK. Evidências científicas sobre os fundamentos do voleibol: importância desse conteúdo para prescrever o treino. Revista Brasileira de Prescrição e Fisiologia do Exercício2013;7(37):78-97.

16. Collet C, Nascimento JV, Ramos V, Stefanello JMF. Construção e validação do instrumento de avaliação do desempenho técnico-tático no voleibol. Revista Brasileira de Cineantropometria e Desempenho Humano2011;13(1):43-51.
17. Rizola Neto, A. Uma proposta de preparação para equipes jovens de Voleibol feminino [dissertação]. Campinas: Universidade Estadual de Campinas - UNICAMP; 2003.

18. Medeiros KR, et al. Bases de dados orçamentários e qualidade da informação: uma avaliação do Finanças do Brasil (Finbra) e do Sistema de Informações sobre Orçamentos Públicos em Saúde (Siops).Revista de Administração Pública 2014;48(5):1113-33.

19. RochaT,et al. Cadastro Nacional de Estabelecimentos de Saúde: evidências sobre a confiabilidade dos dados. Ciência \& Saúde Coletiva2018;23(1):229-40. 